\title{
A Stochastic Unit Commitment Model for Integrating Renewable Supply and Demand Response
}

\author{
Anthony Papavasiliou \\ Department of Industrial Engineering and \\ Operations Research \\ University of California at Berkeley \\ Berkeley, CA 94709 \\ Email: tonypap@berkeley.edu
}

\author{
Shmuel S. Oren \\ Department of Industrial Engineering and \\ Operations Research \\ University of California at Berkeley \\ Berkeley, CA 94709 \\ Email: oren@ieor.berkeley.edu
}

\begin{abstract}
We present a stochastic unit commitment model for assessing the reserve requirements resulting from the large-scale integration of renewable energy sources and deferrable demand in power systems. We present three alternative demand response paradigms for assessing the benefits of demand flexibility in absorbing the uncertainty and variability associated with renewable supply: centralized co-optimization of generation and demand by the system operator, demand bids and coupling renewable resources with deferrable loads. We present simulation results for a model of the Western Interconnection.
\end{abstract}

\section{INTRODUCTION}

This paper focuses on assessing the benefits of demandside flexibility on absorbing the variability and uncertainty of renewable supply. In the paper we consider three fundamental approaches for modeling flexible demand. At the fully centralized end, we consider the case where the system operator centrally co-optimizes the dispatch of demandside resources, renewable supplies and generators. This is unrealistic in practice as the system operator operates the system at a bulk scale and cannot enforce control on the system down to the retail level. In addition, the optimization problem at hand is too complex to solve. Nevertheless, this ideal model provides a benchmark for the potential benefits of demand flexibility. Sioshansi [1] considers this model in a deterministic setting. We extend this approach to account for the uncertainty introduced by renewable energy supply. A fully decentralized approach for coordinating demand response that we also consider in this paper is to establish real-time pricing at the retail level. This possibility was introduced by Schweppe et al. [2] and is discussed by Borenstein et al. [3]. The common approach of reasoning about real-time pricing in the power system economics literature is the use of decremental demand bids. Sioshansi and Short [4] use this approach in the context of a unit commitment model. Borenstein and Holland [5] and Joskow and Tirole [6], [7] also use this approach for analyzing retail pricing. However, there is strong institutional opposition to this approach as it exposes retail consumers to the volatility of electricity prices. In addition, real-time prices often fail to convey the economic value of demand response due to the non-convex operating costs of system operations. This effect has been reported by Sioshansi [4]. Moreover, demand-side bidding fails to capture the cross-elasticity of deferrable demand over time. An intermediate approach for integrating demand response that we consider in this paper is coupling the operations of renewable resources with deferrable demand. The motivation of coupling renewable generation with deferrable demand is to create a net resource that appears "behind the meter" as a virtual power plant from the point of view of the system operator.

In order to accurately assess the impacts of renewable energy and demand response integration on power system operations it is necessary to represent the balancing operations of the remaining grid by using a unit commitment model of the daily scheduling and dispatch procedure performed by the system operator. In this paper, a stochastic formulation of the unit commitment model is used in order to quantify the level of reserves that are required in order to integrate renewable resources reliably and the contribution of demand response in mitigating these requirements. The fact that a unit commitment model can accurately represent the balancing operations of the system has resulted in numerous renewable integration studies based on unit commitment, including Ruiz et al. [8], Sioshansi and Short [4], Wang et al. [9], Contantinescu et al. [10], Tuohy et al. [11], Morales et al. [12], Bouffard et al. [13], Papavasiliou et al. [14] and Papavasiliou and Oren [15].

Despite the fact that stochastic unit commitment is appropriate for quantifying the impacts of renewable energy and demand response integration, the model introduces challenges in terms of representing uncertainty and solving the resulting large-scale mixed integer linear program. Dupacova et al. [16] pioneered scenario selection and scenario reduction algorithms motivated by stability results on the optimal values of stochastic programs with respect to perturbations in probability measures. Faster variants of these algorithms were presented by Heitsch et al. [17] and their effectiveness in the stochastic unit commitment problem was demonstrated by Gröwe-Kuska 
et al. [18]. As a result of this work, this class of algorithms was subsequently adopted in the stochastic unit commitment literature. Among the wind integration studies referenced above, the algorithms of Heitsch et al. [17] are used by Tuohy et al. [11] and Morales et al. [12]. Although these algorithms can be applied in a straightforward fashion for the case of renewable integration studies without transmission constraints, they may underestimate the severity of certain scenarios [14]. Furthermore, it is not clear how they can be used for selecting and weighing scenarios of multi-area renewable production multiplexed with composite element (generator and transmission line) outages [15]. In order to address these challenges, Papavasiliou and Oren [15] propose a scenario selection and weighing algorithm inspired by important sampling that is also used in this paper. According to the proposed algorithm, scenarios are selected according to their effect on the expected cost and weighed such that their selection does not bias the objective function of the stochastic unit commitment formulation. In order to address the size of the resulting large-scale mixed integer linear program, the decomposable structure of the problem can be exploited. Decomposition algorithms based on Lagrangian relaxation for the stochastic unit commitment problem were pioneered by Takriti et al. [19]. Alternative relaxations were subsequently presented by Carpentier et al. [20] and Nowak and Römisch [21]. Shiina and Birge [22] presented an alternative decomposition approach for solving the stochastic unit commitment problem using column decomposition. In Papavasiliou et al. [14] the authors present a dual decomposition algorithm for solving the problem that is also used in this paper.

The remaining paper is organized as follows. In Section II we provide an overview of the components of our model. In Section III we describe in detail the demand flexibility models that we consider in our analysis. Results from a test case of the Western Interconnection are presented in Section IV. In Section V we discuss the conclusions of our work.

\section{MOdEL OVERVIEW}

The modeling approach adopted in this paper follows a two-stage stochastic formulation proposed by Ruiz et al. [23]. Generators are partitioned in a set of slow resources that need to be committed in the day-ahead time frame and a set of fast generators that can be committed and dispatched in real time, after uncertainty in the system has been revealed. In Fig. 1 we present a diagram for integrating demand response models with the unit commitment and real-time dispatch model. The decision support module is a stochastic unit commitment model that determines the day-ahead unit commitment schedule of slow generation resources, while accounting for the randomness of renewable supply and firm (inflexible) demand. Given the day-ahead schedule determined by the stochastic unit commitment model, we evaluate the performance of various demand response strategies in the economic dispatch phase, represented by the evaluation module.

\section{A. Statistical Models}

The analysis is driven by uncertainty in renewable supply, firm (inflexible) demand, and real-time prices. We use a second order autoregressive time series model for representing wind speed. A static power curve is used for converting wind speed to wind power production. Our calibration and simulation methodology follows Brown et al. [24], Torres et al. [25] and Callaway [26]. The calibration and simulation procedure follows the steps outlined in Papavasiliou and Oren [15]. The fit of the wind model to the available data is presented in Papavasiliou and Oren [15]. Firm demand is also modeled as a second order autoregressive process, assume to be independent of renewable production.

\section{B. Stochastic Unit Commitment}

In order to determine the day-ahead reserves that are committed by the system operator in order to accommodate the simultaneous integration of renewable supply and deferrable demand, we formulate a unit commitment model that assumes that the system operator co-optimizes the dispatch of flexible loads and generation resources. The model follows the formulation in Papavasiliou et al. [14]. An integral constraint can be introduced to the stochastic unit commitment model that represents the need to supply a total of $R$ units of energy to deferrable loads within the horizon $T$ :

$$
\sum_{t \in T} e_{s t}=R, s \in S,
$$

where $S$ is the set of scenarios and $e_{s t}$ is the amount of energy supplied to deferrable loads in scenario $s$, period $t$.

\section{DEMAND FLEXIBILITY}

As we discuss in Section I, we consider three fundamental approaches for modeling demand flexibility. In a fully decentralized approach, price-responsive loads bid valuations and demand flexibility is introduced in the objective function of the problem. Decision variables for such price-responsive loads are denoted by $d_{l t}$. In a fully centralized approach, demand flexibility can be accounted for explicitly by the system operator and is introduced in the problem through constraints rather than through the objective function. In this case the system operator controls loads directly through decision variable $e_{t}$ while respecting their operating constraints. Coupling represents an intermediate approach where deferrable loads coordinate their consumption with renewable suppliers in order to appear "behind the meter" from the point of view of the system operator.

\section{A. Centralized Load Control}

In the centralized load control approach we assume that the system operator co-optimizes the dispatch of flexible loads and generation resources. The formulation of the centralized load control model is obtained from the economic dispatch model by enforcing $d_{l t}=0$ for all loads $l$ and periods $t$. The net demand $D_{s t}$ in the market clearing constraint represents the difference of random firm demand and random renewable supply. 


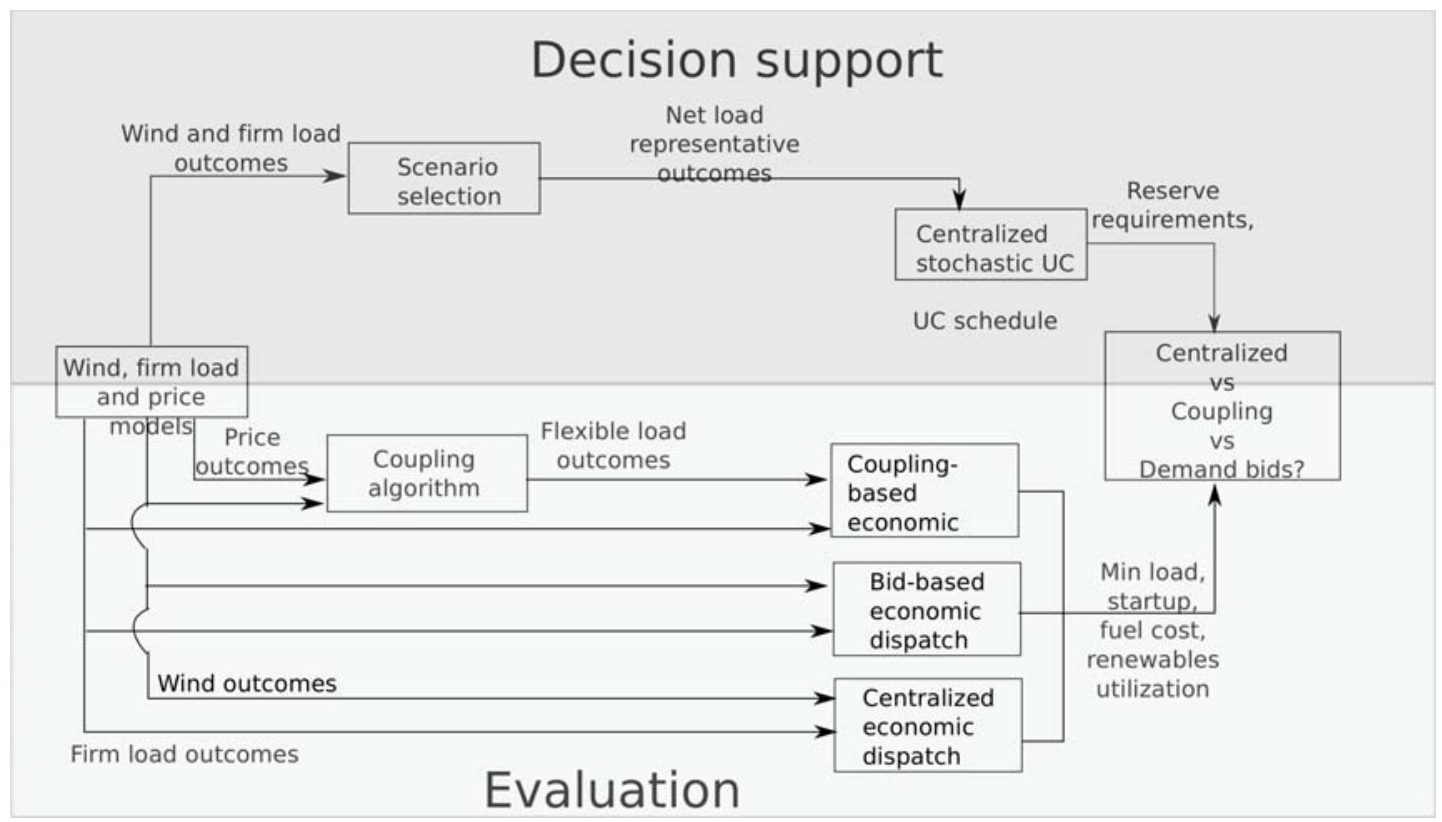

Fig. 1. Overview of the model.

\section{B. Demand Bids}

The demand model that we present in this section is based on Borenstein and Holland [5] and Joskow and Tirole [6], [7]. We assume a linear demand function that consists of a fraction $\alpha$ of inflexible consumers who face a fixed retail price $\lambda^{R}$, and a fraction $1-\alpha$ of price-responsive consumers who face the real-time price of electricity $\lambda_{t}$. The demand function $D_{t}(\cdot)$ for each period can therefore be expressed as:

$$
D_{t}\left(\lambda_{t} ; \omega\right)=a_{t}(\omega)-\alpha b \lambda^{R}-(1-\alpha) b \lambda_{t},
$$

where $\omega$ represents an element of the sample space that determines the realized inflexible demand, $a_{t}(\omega)$ is the intercept and $b$ is the slope of the demand function. Note that we assume a common slope for all time periods and a timevarying stochastic intercept that depends on the realization of inflexible demand. The calibration of the demand functions is described in detail in Papavasiliou [27]. The resulting economic dispatch model is obtained by enforcing $e_{t}=0$ in the economic dispatch model. Again, net demand in the market clear ing constraint represents the difference of firm demand and renewable supply.

\section{Coupling}

In this model an aggregator contractually owns the output from a large group of renewable generation assets. The aggregator enters into a contractual agreement to supply deferrable loads. Loads specify their energy demand in the form of requests for a certain amount of energy over a fixed time window. The aggregator can control the loads directly and uses renewable power from its assets as the primary energy source for satisfying deferrable demand. In the case of renewable supply shortage, the aggregator can resort (to a limited extent) to the real-time market for procuring power at the prevailing real-time price. The aggregator compensates deferrable loads at a rate $\rho$ for each unit of unserved energy. Any excess renewable power is supplied to the system. The setup is similar to dynamic scheduling [28], whereby demand and supply resources from different control areas pair their schedules in order to produce a zero net output to the remaining system. Such scheduling is currently implemented in the ERCOT market. The model is described in detail in Papavasiliou and Oren [29].

\section{RESULTS}

\section{A. Preliminaries}

We present results for a model of the Western Electricity Coordinating Council (WECC), also used in other studies [30], [14], [15]. The model consists of 124 generators and we do not account for transmission constraints. Details about the system can be found in Papavasiliou et al. [14]. We consider three wind/demand response integration studies that are summarized in Table I. For each level of wind integration, we assume a demand response integration level that is approximately onefor-one in terms of energy demand and capacity. We assume that deferrable requests span over 24 hours. We consider 6 levels of power supply for the control problem. The penalty of unserved energy is $\rho=5000 \$ / \mathrm{MWh}$. We use 12 scenarios for the formulation of the stochastic unit commitment model. The wind data that is used for the calibration of the statistical models is based on the National Renewable Energy Laboratory (NREL) 2006 Western Wind and Solar Integration Study [31]. The moderate and deep wind integration studies correspond to the 2012 and 2020 wind integration targets of California. Further details about the wind production data can 
TABLE I

KEY PARAMETERS OF THE DEMAND RESPONSE CASE STUDY.

\begin{tabular}{|l|r|r|r|}
\hline & No Wind & Moderate & Deep \\
\hline Wind capacity (MW) & 0 & 6688 & 14143 \\
DR Capacity C (MW) & 0 & 5000 & 10000 \\
Daily wind energy (MWh) & 0 & 46485 & 95414 \\
Daily DR energy $R(\mathrm{MWh})$ & 0 & 40000 & 80000 \\
Flexible/firm demand (\%) & 0 & 6.1 & 12.2 \\
\hline
\end{tabular}

be found in Papavasiliou and Oren [15]. In order to reduce the computational requirements of the model we focus on eight representative day types instead of simulating an entire year of operations for the system.

\section{B. Costs, Load Loss, Capacity Requirements and Spillage}

In table II we present the operating costs and daily load losses for the case with no wind and no demand response in the system. The operating costs do not include the cost of lost load. Note that for the average demand of the system under consideration, the 1-day-in-10-years reliability criterion requires daily load shed of no more than $179 \mathrm{MWh}$. This can be used as a benchmark against which we can compare the extent to which each demand response mechanism is acceptable from a reliability perspective.

In Tables III, V we present the daily operating cost of each policy for the moderate and deep integration cases respectively. The column with bold figures, that corresponds to centralized load dispatch by the system operator, contains absolute cost values. Cost figures corresponding to the other policies are relative to the centralized operating costs. The row with total costs weighs the cost of each day type with its relative frequency in the year in order to yield annual results. The last row shows the relative performance of centralized control with respect to the other policies, normalized by the cost of centralized control. Note that the operating costs of pricebased demand response outperform those of coupling. This can be attributed to the diversification effect of including flexible demand in the market. The "cost of anarchy" that results from using price signals in order to control load response, rather than centralized control, ranges from $2.43 \%-6.88 \%$ for the case of demand-side bidding and $3.06 \%-8.38 \%$ in the case of coupling. Although demand bids result in lower operating costs, demand-side bidding results in load shedding that is 3.4 times greater than the 1-day-in-10-years criterion for the moderate integration case and 6.8 times greater for the deep integration case. Coupling results in the operation of the system within reliability limits as we note in Tables IV, VI.

In Table VII we present a breakdown of operating costs by type for each of the policies that we consider for each integration level. We note that price response and coupling result in cost increases in all cost categories. As Sioshansi [1] argues, the marginal cost signal itself does not necessarily induce efficient load response due to the fact that it fails to capture the non-convex operating costs of the system. The observation of Sioshansi is also supported by our results.

In Table VIII we present the amount of capacity that is committed by each policy as well as the amount of renew-
TABLE II

DAILY COST OF OPERATIONS AND LOAD SHEDDING FOR EACH DAY TYPE FOR THE DEMAND RESPONSE STUDY - NO WIND.

\begin{tabular}{|l|r|r|}
\hline & Daily Cost (\$) & Shed (MWh) \\
\hline WinterWD & $7,390,206$ & 0.001 \\
SpringWD & $7,145,737$ & 4.317 \\
SummerWD & $13,684,880$ & 30.869 \\
FallWD & $9,589,506$ & 0 \\
WinterWE & $6,079,003$ & 0.001 \\
SpringWE & $5,855,883$ & 0 \\
SummerWE & $11,839,573$ & 0 \\
FallWE & $7,868,146$ & 154.285 \\
\hline Total & $9,012,031$ & 17.301 \\
\hline
\end{tabular}

TABLE III

DAILY COST OF OPERATIONS FOR EACH DAY TYPE FOR THE DEMAND RESPONSE STUDY - MODERATE INTEGRATION.

\begin{tabular}{|l|r|r|r|}
\hline & \multicolumn{1}{|c|}{$\begin{array}{c}\text { Cost (\$) } \\
\text { Centralized }\end{array}$} & $\begin{array}{c}\Delta \text { Cost (\$) } \\
\text { Coupled }\end{array}$ & $\begin{array}{c}\Delta \text { Cost (\$) } \\
\text { Decoupled }\end{array}$ \\
\hline WinterWD & $\mathbf{7 , 3 2 0 , 6 2 0}$ & 256,740 & 300,051 \\
SpringWD & $\mathbf{6 , 4 0 8 , 3 5 5}$ & 172,006 & 139,589 \\
SummerWD & $\mathbf{1 3 , 6 2 5 , 1 3 6}$ & 155,096 & 219,124 \\
FallWD & $\mathbf{9 , 6 4 0 , 0 1 7}$ & 316,089 & 157,159 \\
WinterWE & $\mathbf{5 , 8 9 0 , 7 5 5}$ & 300,701 & 246,408 \\
SpringWE & $\mathbf{3 , 6 3 7 , 2 4 0}$ & 707,223 & 244,353 \\
SummerWE & $\mathbf{1 1 , 7 3 9 , 1 7 7}$ & 176,230 & 234,101 \\
FallWE & $\mathbf{7 , 7 3 5 , 5 0 2}$ & 277,817 & 189,465 \\
\hline Total & $\mathbf{8 , 6 7 7 , 8 5 7}$ & 265,128 & 211,010 \\
\hline relative $(\%)$ & & 3.06 & 2.43 \\
\hline
\end{tabular}

TABLE IV

DAILY LOAD LOSS FOR EACH DAY TYPE FOR THE DEMAND RESPONSE STUDY - MODERATE INTEGRATION.

\begin{tabular}{|l|r|r|r|}
\hline & $\begin{array}{r}\text { Shed (MWh) } \\
\text { Centralized }\end{array}$ & $\begin{array}{c}\text { Shed (MWh) } \\
\text { Coupled }\end{array}$ & $\begin{array}{c}\text { Shed (MWh) } \\
\text { Decoupled }\end{array}$ \\
\hline WinterWD & 0 & 0 & 177.257 \\
SpringWD & 1.532 & 1.869 & 701.828 \\
SummerWD & 3.617 & 4.346 & 821.719 \\
FallWD & 1.661 & 1.661 & 799.323 \\
WinterWE & 0 & 0 & 642.105 \\
SpringWE & 0 & 0.249 & 453.791 \\
SummerWE & 0.059 & 1.100 & 215.816 \\
FallWE & 6.792 & 10.005 & 976.766 \\
\hline Total & 1.705 & 2.217 & 609.914 \\
\hline
\end{tabular}

TABLE V

DAILY COST OF OPERATIONS FOR EACH DAY TYPE FOR THE DEMAND RESPONSE STUDY - DEEP INTEGRATION.

\begin{tabular}{|l|r|r|r|}
\hline & $\begin{array}{c}\text { Cost (\$) } \\
\text { Centralized }\end{array}$ & $\begin{array}{c}\Delta \text { Cost (\$) } \\
\text { Coupled }\end{array}$ & $\begin{array}{c}\Delta \text { Cost (\$) } \\
\text { Decoupled }\end{array}$ \\
\hline WinterWD & $\mathbf{6 , 6 5 6 , 6 6 5}$ & 633,164 & 556,775 \\
SpringWD & $\mathbf{5 , 6 9 2 , 8 6 0}$ & 978,182 & 572,465 \\
SummerWD & $\mathbf{1 3 , 6 6 1 , 8 6 2}$ & 505,869 & 835,609 \\
FallWD & $\mathbf{9 , 3 2 1 , 2 8 1}$ & 772,659 & 404,523 \\
WinterWE & $\mathbf{5 , 2 2 0 , 1 0 9}$ & 711,882 & 616,931 \\
SpringWE & $\mathbf{4 , 2 5 1 , 6 0 0}$ & 910,253 & 576,010 \\
SummerWE & $\mathbf{1 2 , 1 3 6 , 2 2 3}$ & 329,929 & 472,930 \\
FallWE & $\mathbf{7 , 9 3 0 , 8 2 3}$ & 700,205 & 515,431 \\
\hline Total & $\mathbf{8 , 4 1 9 , 3 2 2}$ & 705,497 & 578,909 \\
\hline relative $(\%)$ & & 8.38 & 6.88 \\
\hline
\end{tabular}

able supply spillage. Capacity requirements do not change significantly for each integration study, which suggests that the additional deferrable demand can be fully absorbed by the installed renewable capacity. Wind spillage is negligible across all cases. 
TABLE VI

DAILY LOAD LOSS FOR EACH DAY TYPE FOR THE DEMAND RESPONSE STUDY - DEEP INTEGRATION.

\begin{tabular}{|l|r|r|r|}
\hline & $\begin{array}{c}\text { Shed (MWh) } \\
\text { Centralized }\end{array}$ & $\begin{array}{c}\text { Shed (MWh) } \\
\text { Coupled }\end{array}$ & $\begin{array}{c}\text { Shed (MWh) } \\
\text { Decoupled }\end{array}$ \\
\hline WinterWD & 0.001 & 8.290 & 552.769 \\
SpringWD & 0 & 351.782 & 1382.459 \\
SummerWD & 0.001 & 36.643 & 1952.332 \\
FallWD & 33.660 & 143.629 & 1210.443 \\
WinterWE & 0 & 0 & 929.960 \\
SpringWE & 0 & 32.601 & 1008.222 \\
SummerWE & 2.081 & 58.725 & 1157.565 \\
FallWE & 57.005 & 132.134 & 1260.137 \\
\hline Total & 10.231 & 112.452 & 1221.492 \\
\hline
\end{tabular}

TABLE VII

BREAKDOWN OF DAILY OPERATING COSTS FOR EACH DEMAND RESPONSE POLICY FOR EACH INTEGRATION LEVEL (\$).

\begin{tabular}{|l|l|l|l|l|}
\hline & Min load & Fuel & Startup & Total \\
\hline \hline No wind & $1,382,156$ & $7,549,491$ & 80,384 & $9,098,537$ \\
\hline Centralized Moderate & $1,246,552$ & $7,364,815$ & 66,489 & $8,677,857$ \\
Bids Moderate & $1,317,383$ & $7,471,363$ & 100,123 & $8,888,866$ \\
Coupled Moderate & $1,330,130$ & $7,532,898$ & 79,958 & $8,942,958$ \\
\hline Centralized Deep & $1,194,606$ & $7,174,611$ & 50,105 & $8,419,322$ \\
Bids Deep & $1,360,543$ & $7,494,472$ & 143,217 & $8,998,232$ \\
Coupled Deep & $1,432,948$ & $7,592,595$ & 99,276 & $9,124,819$ \\
\hline
\end{tabular}

TABLE VIII

CAPACITY REQUIREMENTS AND WIND POWER SPILLAGE FOR EACH DEMAND RESPONSE POLICY.

\begin{tabular}{|l|l|l|}
\hline & Capacity (MW) & Spillage (MWh) \\
\hline No wind & 26,123 & N/A \\
Moderate & 26,254 & 0 \\
Deep & 26,789 & 2 \\
\hline
\end{tabular}

\section{CONCLUSIONS}

In this paper we present a stochastic unit commitment model that accounts for renewable energy and demand response integration. We compare three demand response paradigms: centralized load dispatch, demand-side bidding and coupling. We analyze the case of no wind in the network, as well as cases of wind integration that correspond to the 2012 and 2020 wind integration targets of California, with a corresponding one-forone increase in flexible demand. Our analysis is performed on a model of the Western Electricity Coordinating Council that consists of 124 generators. We find that the "cost of anarchy" incurred by decentralizing demand response ranges between $3.06 \%-8.38 \%$ for the case of coupling. Price response results in a cost increase ranging between $2.43 \%-6.88 \%$ of the cost corresponding to centralized load dispatch. However, price response fails to capture the cross-elasticity of demand across time periods, resulting in excessive lost load. For the case studies that we consider, the additional integration of deferrable demand imposes no additional capacity requirements to the system.

\section{ACKNOWLEDGMENTS}

This research was funded by NSF Grant IIP 0969016, the US Department of Energy through a grant administered by the Consortium for Electric Reliability Technology Solutions
(CERTS), the Siemens Corporation under the UC Berkeley CKI initiative and the Federal Energy Regulatory Commission.

\section{REFERENCES}

[1] R. Sioshansi, "Modeling the impacts of electricity tariffs on plug-in hybrid electric vehicle charging, costs and emissions," submitted to Operations Research, 2011.

[2] F. C. Schweppe, M. C. Caramanis, R. D. Tabors, and R. E. Bohn, Spot Pricing of Electricity. Kluwer Academic Publishers, 1988.

[3] S. Borenstein, M. Jaske, and A. Rosenfeld, "Dynamic pricing, advanced metering and demand response in electricity markets," University of California Energy Institute, Tech. Rep., October 2002.

[4] R. Sioshansi and W. Short, "Evaluating the impacts of real time pricing on the usage of wind power generation," IEEE Transactions on Power Systems, vol. 24, no. 2, pp. 516-524, May 2009.

[5] S. Borenstein and S. Holland, "On the efficiency of competitive electricity markets with time-invariant retail prices," RAND Journal of Economics, vol. 36, no. 3, pp. 469-493, Autumn 2005.

[6] P. Joskow and J. Tirole, "Retail electricity competition," RAND Journal of Economics, vol. 37, no. 4, pp. 799-815, Winter 2006.

[7] _ "Reliability and competitive electricity markets," RAND Journal of Economics, vol. 38, no. 1, pp. 60-84, Spring 2007.

[8] P. A. Ruiz, R. C. Philbrick, and P. W. Sauer, "Wind power dayahead uncertainty management through stochastic uc policies," in Power Systems Conference and Exposition, March 2009, pp. 1-9.

[9] J. Wang, M. Shahidehpour, and Z. Li, "Security-constrained unit commitment with volatile wind power generation," IEEE Transactions on Power Systems, vol. 23, no. 3, pp. 1319-1327, August 2008.

[10] E. M. Constantinescu, V. M. Zavala, M. Rocklin, S. Lee, and M. Anitescu, "A computational framework for uncertainty quantification and stochastic optimization in unit commitment with wind power generation," IEEE Transactions on Power Systems, vol. 26, no. 1, pp. 431-441, February 2011.

[11] A. Tuohy, P. Meibom, E. Denny, and M. O’Malley, "Unit commitment for systems with high wind penetration," IEEE Transactions on Power Systems, vol. 24, no. 2, pp. 592-601, May 2009.

[12] J. M. Morales, A. J. Conejo, and J. Perez-Ruiz, "Economic valuation of reserves in power systems with high penetration of wind power," IEEE Transactions on Power Systems, vol. 24, no. 2, pp. 900-910, May 2009.

[13] F. Bouffard and F. D. Galiana, "Stochastic security for operations planning with significant wind power generation," IEEE Transactions on Power Systems, vol. 23, no. 2, pp. 306-316, May 2008.

[14] A. Papavasiliou, S. S. Oren, and R. P. O'Neill, "Reserve requirements for wind power integration: A scenario-based stochastic programming framework," IEEE Transactions on Power Systems, vol. 26, no. 4, pp. 2197-2206, November 2011.

[15] A. Papavasiliou and S. S. Oren. (2011) Multi-area stochastic unit commitment for high wind penetration in a transmission constrained network. [Online]. Available: http://www3.decf.berkeley.edu/ tonypap/ scengentransv6.pdf

[16] J. Dupacova, N. Gröwe-Kuska, and W. Römisch, "Scenario reduction in stochastic programming: An approach using probability metrics," Math Programming, vol. 95, no. 3, pp. 493-511, 2003.

[17] H. Heitsch and W. Römisch, "Scenario reduction algorithms in stochastic programming," Computational Optimization and Applications, vol. 24, no. 2-3, pp. 187-206, 2003.

[18] N. Gröwe-Kuska, K. C. Kiwiel, M. P. Nowak, W. Romisch, and I. Wegner, Decision Making Under Uncertainty: Energy and Power, ser. IMA Volumes in Mathematics and Its Applications. New York: Springer-Verlag, 2002, vol. 128, ch. Power Management in a HydroThermal System Under Uncertainty by Lagrangian Relaxation, pp. 3970.

[19] S. Takriti, J. R. Birge, and E. Long, "A stochastic model for the unit commitment problem," IEEE Transactions on Power Systems, vol. 11, no. 3, August 1996

[20] P. Carpentier, G. Cohen, J.-C. Culioli, and A. Renaud, "Stochastic optimization of unit commitment: a new decomposition framework," IEEE Transactions on Power Systems, vol. 11, no. 2, pp. 1067-1073, May 1996.

[21] M. P. Nowak and W. Römisch, "Stochastic lagrangian relaxation applied to power scheduling in a hydro-thermal system under uncertainty," Annals of Operations Research, vol. 100, no. 1-4, pp. 251-272, 2000. 
[22] T. Shiina and J. R. Birge, "Stochastic unit commitment problem," International Transactions on Operations Research, vol. 11, no. 95, pp. 19-32, 2004.

[23] P. A. Ruiz, R. C. Philbrick, E. Zack, K. W. Cheung, and P. W. Sauer, "Uncertainty management in the unit commitment problem," IEEE Transactions on Power Systems, vol. 24, no. 2, pp. 642-1651, May 2009.

[24] B. G. Brown, R. W. Katz, and A. H. Murhpy, "Time series models to simulate and forecast wind speed and wind power," Journal of Climate and Applied Meteorology, vol. 23, pp. 1184-1195, 1984.

[25] J. L. Torres, A. Garcia, M. D. Blas, and A. D. Francisco, "Forecast of hourly wind speed with ARMA models in Navarre (Spain)," Solar Energy, vol. 79, no. 1, pp. 65-77, July 2005.

[26] D. Callaway, "Sequential reliability forecasting for wind energy: Temperature dependence and probability distributions," IEEE Transactions on Energy Conversion, vol. 25, pp. 577-585, June 2010.

[27] A. Papavasiliou, "Coupling renewable energy supply with deferrable demand," Ph.D. dissertation, U.C. Berkeley, October 2011.

[28] E. Hirst and B. Kirby, "Ancillary-service details: Dynamic scheduling," Oak Ridge National Laboratory, Tech. Rep., January 1997.

[29] A. Papavasiliou and S. S. Oren, "Integration of contracted renewable energy and spot market supply to serve flexible loads," in 18th World Congress of the International Federation of Automatic Control, August 2011.

[30] N.-P. Yu, C.-C. Liu, and J. Price, "Evaluation of market rules using a multi-agent system method," IEEE Transactions on Power Systems, vol. 25, pp. 470-479, February 2010.

[31] R. Piwko, K. Clark, L. Freeman, G. Jordan, and N. Miller, "Western wind and solar integration study," National Renewable Energy Laboratory, Tech. Rep., May 2010. 